\title{
Incurred sample reproducibility: views and recommendations by the European Bioanalysis Forum
}

Following intensive discussions, review, alignment of procedures and multiple surveys among their member companies, the European Bioanalysis Forum (EBF) is providing a recommendation on how to integrate incurred sample reproducibility (ISR) in the bioanalytical process. The recommendation aims to provide guidance throughout the lifecycle of a validated method, including the application of the method in study support. In its recommendation, the EBF considers both the internal discussions with EBF member companies, as well as the input provided in international meetings where ISR was discussed. The ultimate goal of the EBF recommendation is to ensure that bioanalytical methods can provide accurate and reproducible concentration data for pharmacokinetic and/or toxicokinetic evaluation, without any compromise, while safeguarding the optimal use of laboratory resources.

Incurred sample reproducibility (ISR) has been on the agenda of bioanalytical scientists for a long time. Regulatory authorities have, on occasion, requested reanalysis of study samples ever since the Shah paper in 1992 [1]. Health Canada (HC) championed these requests for bioavailability and bioequivalence (BE) studies throughout the 1990s [101], until they removed this regulatory requirement in 2003 [102]. Surprisingly, only shortly after $\mathrm{HC}$ moved away from ISR, the US FDA Centre for Drug Evaluation (CDER) put it on the calendar again. One of the reasons, as mentioned by the FDA in several meetings, was the observation made in some filings that some pharmacokinetic (PK) repeats, or unintended repeats in multi-analyte assays, resulted in second values being as much as 300\% different from their original reported values. At several regional and international bioanalytical conferences, the subject was rediscussed. Some good examples of these meetings or discussions groups are Applied Pharmaceutical Analysis (APA) from Boston Society for Advanced Therapeutics (BSAT), Delaware Valley Drug Metabolism Discussion Group (DVDMDG) meetings and the Canadian Calibration and Validation Group (CVG) workshops on regulated bioanalysis. Going forward, the FDA emphasized that ISR had to become an integral aspect of bioanalytical support in preclinical and, more importantly, clinical studies. Intensive discussions with all partners in industry resulted in a number of publications and recommendations [2-4,103,104]. Also, within the European Bioanalysis Forum
$(\mathrm{EBF})$, an organization of bioanalytical scientists from European or global pharma companies with bioanalysis teams active in Europe, the need and added value of regular reanalysis of incurred samples was discussed extensively on several occasions - these included internal discussions with member companies as well as discussions in EBF-organized or EBF-attended external meetings. Several surveys were organized to share results and experiences on the subject of ISR. This article provides detailed feedback of these discussions and experience and summarizes the EBF's recommendation towards the bioanalytical community on ISR.

\section{EBF: background}

In 2006, 12 European pharmaceutical companies founded the EBF. At the early meetings, the initial focus of the discussions was on the issues of ISR, matrix suppression and metabolite quantification. As an organization, the EBF's goal is to share, discuss and potentially optimize or seek alignment on a broad array of bioanalytical topics including science, procedures, business tools and technology, and regulatory issues. Open discussion and alignment, where appropriate or possible, is enabling the member companies to optimize their internal bioanalytical processes. To date, the organization counts 27 member companies and has recently also formed an interest group on macromolecules (EBF-IGM) with focus on special needs related to the development, validation and application of ligand-binding assays (LBAs) and immunogenicity assessment.
Philip Timmerman'†, Silke Luedtke², Peter van Amsterdam ${ }^{3}$, Margarete

Brudny-Kloeppel ${ }^{4}$ \& Berthold Lausecker ${ }^{5}$ ${ }^{\dagger}$ Author for correspondence 'Johnson \& Johnson, Turnhoutseweg 30, B-2340

Beerse, Belgium

Tel.: +32 14603581

Fax: +32 14605। 10

E-mail: ptimmerm@ its.jnj.com

${ }^{2}$ Boehringer-Ingelheim ${ }^{3}$ Solvay Pharmaceutical ${ }^{4}$ Bayer Schering Pharma AG

${ }^{5} \mathrm{~F}$. Hoffmann-La Roche 


\section{Contributors, other EBF member companies: \\ Stephanie Fischmann ${ }^{6}$, Susanne Globig7, Carl-Johan Sennbro ${ }^{8}$, Josep M Jansat', Hans Mulder'10, Elizabeth Thomas", Magnus Knutsson'2, Dirk Kasel'3, \\ Stephen A White ${ }^{14}$, Morten Anders Kall'5, Nathalie \\ Mokrzycki-Issartel' ${ }^{16}$, Achim Freisleben ${ }^{17}$, Fernando Romero ${ }^{18}$, Michael Pilgård Andersen ${ }^{19}$, Norbert Knebel ${ }^{20}$, Marcel de Zwart ${ }^{21}$, Sirpa Laakso ${ }^{22}$, Richard S Hucker ${ }^{23}$, Dietmar Schmidt ${ }^{24}$, Ben Gordon ${ }^{25}$, \\ ${ }^{7}$ Actelion Pharmaceuticals Ltd \\ ISR: background \\ The recent renewed interest in ISR and its inclu- sion in bioanalytical support originate from observations in industry as well as observations made by the FDA during audits or reviews. In some studies, repeat analysis of incurred samples yielded significantly different concentrations when compared with the initial values. This observation was based on differences between original and repeat values, resulting from dupli- cate analytical runs, which both passed the acceptance criteria based on calibration and qual- ity-control (QC) samples. As this also occurred in bioequivalence studies, it added to an increased concern by the FDA regarding the validity of bio- analytical methods during routine application. Moreover, in some of the early cases observed by the FDA, industry failed to provide adequate explanation or follow-up on these anomalous data. More recently, root cause analysis of some interesting cases was presented and discussed at multiple international meetings sponsored by the FDA, American Association of Pharmaceutical Scientists (AAPS), BSAT, CVG, EBF and other business partners.}

${ }^{8}$ Active Biotech

'Laboratorios Almirall SA

${ }^{10}$ Astellas

"AstraZeneca

${ }^{12}$ Ferring Pharmaceuticals

${ }^{13} \mathrm{Grünenthal} \mathrm{GmbH}$

${ }^{14} \mathrm{Glaxo}$ SmithKline

${ }^{15} \mathrm{H}$ Lundbeck A/S

16Merck\&Co

${ }^{17}$ Merck Serono

${ }^{18}$ Novartis Pharma AG

${ }^{19}$ Novo Nordisk

${ }^{20}$ Nycomed

${ }^{21}$ Schering-Plough

${ }^{22}$ Orion Corporation Orion

Pharma

${ }^{23}$ Pfizer

${ }^{24}$ Sanofi-Aventis

${ }^{25}$ Servier

${ }^{26}$ Shire Pharmaceuticals

${ }^{27}$ UCB Pharma

\section{Connecting ISR \& method validation}

Method development and subsequent method validation, as prescribed in FDA's guidance [5], have a number of known limitations. One of them is that, as per the guidance, a method needs to be validated prior to the analysis of study samples with that particular method. As a consequence, human methods were certainly considered validated without ever having been applied to study samples. Although calibrators or QC samples are intended to mimic the incurred samples as closely as possible, these spiked samples might not be representative with respect to the robustness or reproducibility of a method when applied to study samples. Recognized areas of difference are intra- and inter-subject and/or patient variability. Examples include fluctuations in concentrations and/or composition of endogenous compounds caused by disease state, presence of (potentially unstable) metabolites and nutritional conditions.

At the 3rd AAPS/FDA Bioanalytical Workshop, held on 1-3 May, 2006 in Arlington, VA, USA, the subject of ISR was one of the major areas of discussion. An early consensus was reflected in the conference report, which was published in 2007 [3]. As stated, this publication did not repeat or contradict the FDA guidance, which remained applicable, but provided additional clarification and recommendations with a view to achieving uniformity among the practitioners and users of quantitative bioanalysis for all types of molecules. On the subject of ISR, the report reflected the consensus within industry of the need and rationale to perform reanalysis of incurred samples in support of the validation of a method or to increase the confidence in the data reported in preclinical or clinical studies. With respect to the implementation, the report left numerous practical issues to the discretion of the bioanalytical scientist, which gradually led to less agreement on these practicalities between the industry and FDA. The most important unresolved areas were the acceptance criteria, the number of reanalyzed samples needed and the frequency of performing ISR (types of studies). In an AAPS workshop, held on 7-8 February, 2008 in Arlington, VA, USA, cosponsored by EBF and other industry organizations, an attempt was made to provide final recommendations and close the discussions on ISR. This workshop yielded more detailed guidance on how to implement ISR, which were initially published on the AAPS website and are summarized in the next paragraph. Recently, the AAPS published the final outcome of the workshop [4]. The additional nuances presented in this paper, which does not cover all aspects mentioned in the published AAPS slide deck, are discussed later.

\section{Highlights of the initial 2008 AAPS/FDA recommendations on ISR - General principles}

- If pharmacokinetics is the primary end point, ISR must be considered on individual samples (e.g., no pooled samples);

- More subjects with fewer samples per subject give a better chance of finding anomalous samples or subjects;

- One sample near $\mathrm{C}_{\max }$ and one near the end of the elimination phase;

- Reporting the data in the study reports (often the FDA does not see the validation reports when they audit a study) does not rule out also including it in the validation report;

- It is necessary to have a standard operating procedure (SOP) or study plan for ISR.

\section{- Incurred sample reanalysis \& failed ISR}

- Incurred sample reproducibility reinforces confidence that a method is valid and reproducible;

- Failed ISR must lead to an investigation; 
- Failed ISR places the bioanalytical portion of the study on hold until an investigation is completed and follow-up action is carried out.

- When to conduct ISR

- Early in the study (but consider needs for laboratory efficiency);

- Not at the end of the study (small toxicokinetics studies may be an exception);

- First in human oncology studies often have intermittent sample receipt, so ISR should be performed as soon as reasonably possible, if appropriate;

- If dose escalation strategy is based on exposure, exercise scientific judgment about how and when to perform ISR;

- The number of times to repeat sample should be consistent with the original method (i.e., if single original assay point, then a single repeat is sufficient).

\section{- Nonclinical good laboratory} practice studies

- Once per species, per method and per laboratory is appropriate;

- Use first subchronic toxicology study for samples;

- Often part of validation activities;

- Follow guidance in workshop/conference report of 2007.

- Clinical studies

- All bioequivalence studies;

- Healthy volunteer studies as appropriate;

- Patient population studies as appropriate;

- Small-molecule drug-drug interaction (DDI) studies as appropriate;

- Disease-state changes in patient population.

\section{Acceptance criteria: ISR}

- Reminder: ISR does not accept or reject a study - failed ISR should lead to investigation and follow-up;

- For small molecules: two thirds of repeats agree within $20 \%$;

- For large molecules: two thirds of repeats agree within 30\%;

- Other approaches for setting acceptance criteria may be equally acceptable.

\section{Further guidance or enhancements provided in the 2009 AAPS meeting report}

The above initial recommendations did not sufficiently consider a number of key questions still outstanding for industry. As an example, recommendation on the acceptance criteria did not specify whether repeat values should be compared with the original value, mean value or other approaches. Later, in other meetings, some additions or changes were proposed by the FDA. Although not stated, a significant number of companies interpreted the acceptance criteria to be designed around the original value - two thirds of repeat values fall within $20 \%$ (30\% for LBAs) of the original value. This was now abandoned and the industry was surprised to find the acceptance criteria change to accept repeat values when two thirds of repeat values fell within $20 \%(30 \%$ for LBAs) of the mean value. Final confirmation of this revised or enhanced vision was presented in the recent AAPS meeting report. At the same time, the report does not mention the initial AAPS suggestion to consider the possibilities of other approaches for setting acceptance criteria.

With respect to the number of samples to be reanalyzed, the FDA and industry were still debating the best way to select samples for ISR. Most companies had already moved away from their initial process to select full PK profiles on a limited number of subjects and were selecting samples based on a 'fewer samples from more subjects' approach (e.g., near $\mathrm{C}_{\max }$ and the lower end of the concentration range). In some instances, sample selection was performed in line with the Rocci paper [6], which, in essence, provided an excellent statistical strategy to limit the number of repeats to approximately $20 \mathrm{sam}$ ples, still making sure that a problem with the method is detected. As a consequence, in most studies, ISR resulted in the reanalysis of anything between 20 and 50 samples per study. In recent communications, the FDA challenged this approach by requesting the repeat of $5-10 \%$ of the study samples as a general rule for small and large studies, respectively, although no guidance is provided on the definition of small and large studies. For these larger studies, this request could amount easily to repetition of 200 or more study samples. The meeting report confirmed the FDA's current thinking on the number of samples to be selected for ISR.

When dealing with the consequences of failed ISR, the initial thinking was that it would place the bioanalytical phase of the study on hold 
until an investigation and follow-up action were completed. Although this was a clear statement, surprisingly, the AAPS meeting report provides relief by considering the possibility of proceeding with the analysis 'at risk'. This statement may need further discussion to understand the consequences.

Finally, the meeting report summarizes a number of topics with no consensus or which were not addressed. As some of them were addressed in the EBF discussions, the current EBF publication can provide additional insight or recommendation.

\section{EBF views \& recommendations on ISR - General considerations}

Presented above are a few of the examples supporting the industry's perception that the ISR target is still moving. From its foundation onwards, EBF stimulated the understanding and harmonized implementation of ISR among its member companies. As already mentioned, the current recommendations on ISR result from several internal discussions with all member companies, discussions in EBF-organized or EBF-attended external meetings, as well as from multiple internal EBF survey results. In addition, the shared and recent EBF experience on ISR, gained in over 500 nonclinical and clinical studies, provided an excellent foundation on which to build. The discussions always aimed at finding the best scientific approach to further optimize the methods and increase the confidence in the reported concentrations. With its recommendations, the EBF intends to provide additional insights and guidance for the industry and to ensure that EBF member companies translate the multiple discussions and publications into one harmonized view. At the same time, the process should foster the scientific and regulatory needs of our work, so that our methods can be validated and applied consistently and efficiently, and also cover the initial intention of ISR: to ensure a validated method is able to measure concentrations in incurred samples for any given study both accurately and reproducibly.

At the same time, additional scientific and procedural insights are provided, aimed at enhancing the practical implementation of ISR in industry.

In principle, the recommendations from the FDA to perform ISR regularly are endorsed by the EBF. Additional clarification and potential enhancements are provided on the overall goal and positioning of ISR in bioanalytical method validation. More specifically, EBF's recommendations focus on the topics for which the discussions and publications provide insufficient detail or clarity or on the potential areas of difference in the vision developed by EBF and these recent publications - examples are the selection of studies, selection of samples, the acceptance criteria and reporting of the ISR data. The EBF recommends that every bioanalytical laboratory should have a SOP or a study plan for ISR.

Incurred sample reproducibility enforces the confidence that a method is valid and reproducible for the intended purpose. Accuracy and precision data generated during method validation using spiked samples should be supplemented with data on repeatability in samples from dosed animals or subjects. As a consequence, ISR can be considered an integral part of method validation. Nevertheless, when considering root cause analysis of failed ISR, it is important to recognize two main categories:

$$
\begin{aligned}
& \text { - Poor methods - ISR as part of method } \\
& \text { validation; } \\
& \text { " Poor execution - ISR as part of a regular } \\
& \text { process check. }
\end{aligned}
$$

Therefore the EBF recommends that laboratories should design the implementation of ISR around the understanding that ISR serves the above two needs.

\section{ISR as part of method validation}

Data on ISR are presented in addition to other critical scientific or procedural features of a validated method as listed below:

- Accuracy/precision;

- Linearity and calibration range (e.g., upper limit of quantification [ULOQ] and lower limit of quantification [LLOQ]);

- Stability (e.g., short-term, long-term, benchtop, freeze/thaw, processed sample, solvent and plasma/blood);

- Matrix effects (for MS-based assays);

- Selectivity towards endogenous compounds (e.g., comedication and metabolites);

- Understanding of metabolism and impact thereof on performance of a particular method.

Well-planned and well-executed ISR will provide scientific documentation of the robustness and repeatability of a method. As a consequence, ISR should preferably be performed early in the lifecycle of a method (upon availability of the first incurred samples for that matrix). Including 
ISR as part of method validation early on will result in better validated methods and should remove the need for frequent re-investigation of ISR in multiple studies.

Like any other critical parameter assessed during method validation, careful consideration should be given regarding the effect of changes to a method and how these changes may affect the validity of the earlier documented ISR for future studies. For that reason, the EBF recommends that ISR should be re-evaluated upon every major method change. SOPs are excellent tools to provide a priori guidance if and when re-assessment of ISR as part of partial method validation is needed.

- ISR as part of regular process check A second reason to perform ISR is to check whether laboratory procedures (e.g., SOPs and analysis protocols) are consistently followed. This interpretation is supported by evaluating the root cause analysis of a number of failed ISRs: inadequate thawing, pipetting errors, insufficient vortexing of samples prior to aliquoting and wrong labelling. None of these are necessarily related to poor methods, but more likely to the analytical scientist's distraction or other similar nonadherence to good practices. In this respect, ISR can be considered a good addition to the inclusion of calibration and QC samples. Since calibration and QC samples are already part of every analytical run and can be considered to cover both their originally intended purpose (verify if the method performs in line with the criteria documented in the validation) and to extend adherence to the processes, they can fulfil the same purpose as ISR in the majority of studies. As a consequence, the EBF does not see a real need to include ISR as a process check in each and every study. Moreover, if ISR was sufficiently investigated during the validation of a method, failing ISR because of poor adherence to processes could be considered to be independent from the method and perhaps more a check of the laboratory or the analytical scientist. As a consequence, EBF feels that 'PK as primary end point', as mentioned in the AAPS recommendations, is not necessarily a good criterion to select studies for ISR.

\section{Frequency of ISR \& selection of studies}

The understanding of the dual purpose of ISR provides excellent guidance for the frequency in which ISR is investigated or the studies for which ISR is needed. Using the criteria considering
ISR as part of method validation, the first time in new matrix (preclinical species or human) or the first time in new target populations are good drivers for identification of studies. These include the first nonclinical study in toxicology species, the first time in humans (evaluate single versus multiple dose), the first time into patients, the application of a method in special populations (e.g., pediatrics, renal or hepatic impairment) and new disease indications (significantly different from the initial therapeutic indication with respect to the impact on sample nature). These examples provide general guidance for the scientific rationale that justifies the ISR process. Similarly, if a certain laboratory performs an assay for the first time and incurred samples are not reanalyzed as part of method transfer and cross validation, ISR should be considered.

Intensive discussions on the need and added value of performing ISR for all DDI work highlighted the limited added scientific value of performing ISR for these studies. It was recognized that investigation and documentation of selectivity or potential matrix effects toward comedicated drugs (and, if needed, their major metabolites) is the main challenge in the analysis of DDI studies. Therefore, no systematic investigation of ISR is recommended for these studies, only regular process checks. Since a lot of these assays are part of the nonproprietary assay lists of contract research organizations (CROs), EBF suggests that CROs, sponsors and regulators work towards a consensus to provide ISR data as part of the method validation data of the DDI assays, rather than in every individual study. This could be supported by historic data on the reproducibility of these assays.

Investigation of ISR in bioequivalence studies is endorsed by the EBF and, although in most cases it can be classified as a process check, the EBF recommends the performance of ISR for all pivotal bioequivalence studies.

In conclusion, the EBF recommends the performance of ISR for the following studies:

- Method validation;

- First time in a new matrix: animal or human;

- The first time in a new target population: first patient study;

- Disease state changes in patient population;

- Upon any major method change (as per SOP);

- First-time use of an existing method in a new laboratory; 


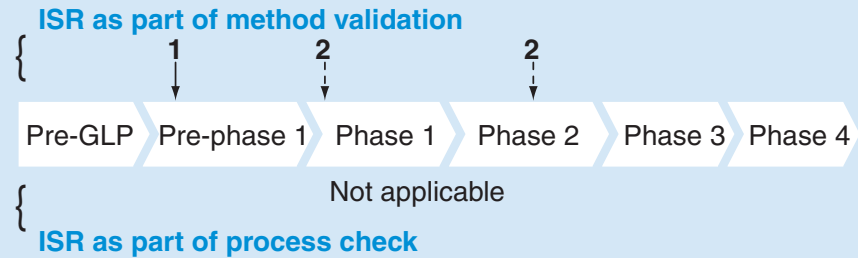

1. First analysis of incurred samples with newly validated method

2. Major method change (as per standard operating procedure)

Figure 1. Incurred sample reproducibility (ISR) as part of method validation and process check for preclinical species.

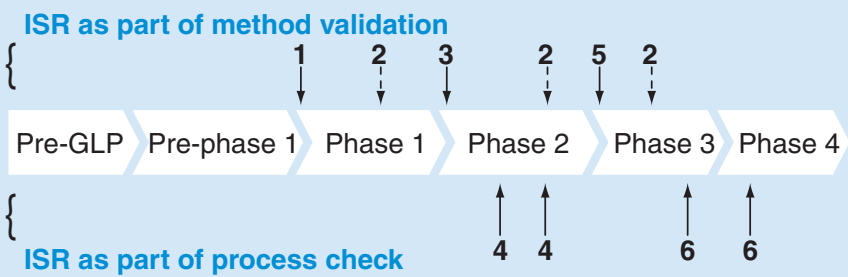

1. First analysis of incurred samples with newly validated method

2. Major method change (as per standard operating procedure)

3. First time into patient population

4. Occasionally for drug-drug interaction studies

5. Special population (guided by scientific rationale)

6. Bioequivalence studies

Figure 2. Incurred sample reproducibility (ISR) as part of method validation and process check for human volunteers and patients.

- Whenever scientific reasons require retesting of ISR (e.g., special populations based on scientific rationale);

- Process check;

- All BE studies;

- Incidental check in any other studies (human PK or DDI study).

Figures I \& $\mathbf{2}$ visualize the above recommended practice.

\section{- Acceptance criteria}

The EBF spent a lot of time working towards harmonized and simple rules for ISR acceptance criteria. In their original assessment, pragmatic acceptance criteria (two thirds of repeat values $30 \%$ or less difference from the initial value [ $40 \%$ for LBAs]) were proposed for the duration of 1 year, during which EBF member companies would gather data on the success or failure of ISR. This approach was presented at the February 2008 AAPS meeting. Although this pragmatic approach was welcomed by numerous participants at the meeting, the proposal received insufficient endorsement from all stakeholders. As a consequence, the EBF revisited and revised their recommendations, producing more stringent acceptance criteria. Industry practice, stimulated by the 2008 workshop outcome, also moved towards the acceptance of two thirds of repeat values less than or equal to $20 \%$ difference (interpreted as from the original value [30\% for LBAs]), being slightly more stringent compared with the EBF's initial proposal. Later, the FDA clarified their expectations as two thirds of values of $20 \%$ or less of the mean value. These latest acceptance criteria are considered by the EBF to move away from the philosophy that the purpose of ISR is to understand the validity of the reported value, which is the original value, where the calculations are based around the difference from the original value and not the mean value. The EBF recommends following these acceptance criteria:

- Two thirds of repeat values within $80-120 \%$ of the original value (chromatographic assays);

- Two thirds of repeat values within 70-130\% of the original value (LBAs).

The EBF has followed up on their intention, expressed at the 2008 AAPS meeting, to monitor the success or failure rate of ISR in studies sponsored by the individual EBF member companies (measured in sponsor's facilities or at CROs). Results were presented at the EBF open meeting in December 2008 [7]. In summary, EBF member companies reported ISR results from over 500 different studies, 220 preclinical and 295 clinical studies, most of them performed in the course of 2008. Approximately 3.5\% (eight out of 220) of preclinical studies had failed ISR, whereas approximately 4\% (12 out of 295) of clinical studies had failed ISR. When analyzing the reasons for failing ISR, there was a 50-50 split between ISR failing due to poor methods or due tohuman error. TABLE I summarizes the results of the combined experience on ISR from the EBF member companies.

\section{- Selection \& number of study samples to be assessed}

With respect to the selection of study samples, we recommend the use of scientific judgment. Multiple subjects and limited samples per subject is a good scientific selection criterion, together with the earlier mentioned Rocci paper [6]. A 5 or $10 \%$ criterion is not seen as a good basis for sample selection, considering the purpose of ISR. Capitalizing on EBF discussions, the above EBF 
philosophy for selection of samples will result typically in 20-50 samples, which, in the majority of cases, will be within the 5 or $10 \%$ range. Taking into account the dual purpose of ISR (to find method flaws and human error), this approach is understood to fit these purposes. Moreover, the fact that ISR serves both the validation of a method and the adherence of the laboratory to sound processes, the latter in addition to the calibration and QC samples, should be taken into account when identifying the number of samples for ISR. As a consequence, reanalyzing hundreds of samples in these large studies to comply with a 5\% criterion is not recommended by the EBF.

\section{- Failed ISR}

The EBF agrees and recommends that any failed ISR must lead to an investigation and that the bioanalytical phase of the study should be placed on hold until an investigation is completed and follow-up action is implemented. Proper documentation of all investigation is warranted. Failed ISR and subsequent investigation/followup actions can lead to reanalysis of all or a part of the study samples or even revalidation of the method. In the latter case, the impact of the failed ISR on other studies in which the previous validated method was used must be assessed and documented.

\section{- Additional considerations}

Pooled versus nonpooled samples

If possible, ISR should be performed on individual samples. Pooled samples should only be considered when ethical considerations prevail. Examples are pediatrics, patients where only limited blood volume can be harvested and very small animals. In cases where pooling is needed, the pooled sample is considered as a new sample and needs to be analyzed twice to obtain an initial value and an ISR value.

\section{Dilutions}

Over-range samples are reanalyzed after dilution to bring them into the linear range of the assay and generate the first value. Upon reanalysis for ISR, the same dilution needs to be maintained. For LBAs, any ISR analysis should always be performed using the same dilution as the first valid analysis.

\section{Samples below the limit of quantification}

The concentration of a selected sample can be below the limit of quantification. On these occasions, it is advisable to reconsider the original
Table 1. Summary of success/failure rate of incurred sample reproducibility*.

$\begin{array}{lll}\text { Parameter } & \text { Preclinical } & \text { Clinical } \\ \text { Number of studies reported } & 220 & 295 \\ \text { Number of studies with failing ISR } & 8 & 12 \\ \begin{array}{l}\text { Failure rate (\%) } \\ \text { Number of ISR failures due to method flaw }\end{array} & 3.6 & 4.1 \\ \begin{array}{l}\text { Number of ISR failures due to human error } \\ \begin{array}{l}\text { Acceptance criteria of the ISR failures at the time of } \\ \text { the experiments: }\end{array}\end{array} & 4 & 7 \\ \quad \text { 4-6-20 on first value } & 4 / 8 & \\ \quad \text { 4-6-20 on mean } & 1 / 8 & 7 / 12 \\ \quad \text { Other } & & 3 / 12 \\ \text { *Data from European Bioanalysis Forum member companies gathered in 2008. } \\ \text { ISR: Incurred sample reproducibility. }\end{array}$

selection and reselect samples with positive values. Any decision should be documented prior to the second analysis.

\section{- Documentation of ISR}

In general, the EBF has no formal recommendation on where to document ISR. Practical considerations need to prevail, providing transparency is ensured. Taking into consideration that ISR is an integral part of method validation, it may be good practice to document or at least cross reference all ISR in the method validation report. However, practical considerations such as reporting templates or agreed SOPs may result in documentation of the ISR results in either the method validation report or the (non)-clinical study reports. For the same reason, ISR performed at sponsor laboaratories or CROs may follow different routes, based on individual company processes. For bioequivalence studies, however, it is the general practice and recommendation to document ISR in the study report.

\section{Conclusion}

In conclusion, the EBF recognizes the need to perform ISR in both clinical and nonclinical studies. However, as an additional clarification and rationale compared with the recent AAPS recommendation, the EBFs position is that, in implementing ISR, consideration should be given to the two major reasons why validated methods fail to deliver reproducible data when a method is applied to incurred samples: poor methods and poor execution of methods. With respect to the first root cause, the EBF recommends documenting ISR early in the lifecycle of a validated method. This should be performed for the method in each matrix and does not remove 
the responsibility of the bioanalytical scientist to take into account all other important parameters that should be tested and documented to obtain accurate and reproducible concentration data. Second, these methods need to be applied in the correct way and for that purpose, it is recommended that ISR is occasionally reinvestigated at later stages in the clinical development of a compound. EBF recommends reassessment of ISR for every important milestone in the lifecycle of a method, especially in the clinical arena (first time in humans, first time in a patient, every major method change and for every final bioequivalence study). However, considering ISR is integrated in method validation from the first validation onwards and repeated at regular intervals, EBF does not see the need to perform ISR in every study. With respect to acceptance criteria, selection of samples and documentation of the ISR experiments, the EBF recommends the use of a slightly different approach compared with the current AAPS recommendation. These small differences in interpretation will not jeopardize the integrity of the ISR in any way, as they are aimed to be both slightly more stringent while allowing more flexibility for the bioanalytical scientist to integrate ISR in the best way possible. Recent benchmark data confirm the low incidence of failed ISR, providing a sound basis for the EBF recommendations.

\section{Disclaimer}

The views expressed in this article are those of the European Bioanalysis Forum and do not necessarily reflect the respective company's position on the subject.

Financial \& competing interests disclosure The authors have no relevant affiliations or financial involvement with any organization or entity with a financial interest in or financial conflict with the subject matter or materials discussed in the manuscript. This includes employment, consultancies, honoraria, stock ownership or options, expert testimony, grants or patents received or pending, or royalties.

No writing assistance was utilized in the production of this manuscript.

\section{Bibliography}

1 Shah VP, Midha KK, Dighe S et al. Analytical methods validation: bioavailability, bioequivalence, and pharmacokinetic studies. Pharm. Res. 9, 588-592 (1992).

2 Savoie N, Booth BP, Bradley T et al. The 2nd CVG workshop on recent issues in good laboratory practice bioanalysis. Bioanalysis 1(1), 19-30 (2009).

3 Viswanathan CT, Bansal s, Booth B et al. Quantitative bioanalytical methods validation and implementation: best practices for chromatographic and ligand binding assays. AAPS J. 9(1), E30-E42 (2007).

4 Fast DM, Kelley M, Viswanathan C et al. AAPS workshop on current topics in GLP bioanalysis: assay reproducibility for incurred samples - implications of crystal city recommendations. AAPS J. 11(2), 238-241 (2009).
5 US FDA. Guidance for industry bioanalytical method validation. US FDA Center for Drug Evaluation and Research Center for Veterinary Medicine (2001).

6 Rocci ML, Devanarayan V, Haughey DB et al. Confirmatory reanalysis of incurred bioanalytical samples. AAPS J. 9(3), E336E343 (2007).

7 Timmerman P, Lausecker B, Abbott R et al. EBF perspective on experience of incurred sample reanalysis. Presented at: EBF Open Symposium. Barcelona, Spain, 2-4 December 2008.

\section{- Websites}

101 Website Health Canada. Conduct and analysis of bioavailability and bioequivalence studies - part B: oral modified release formulations www.hc-sc.gc.ca/dhp-mps/prodpharma/ applic-demande/guide-ld/bio/bio-b-eng.php

102 Website Health Canada. Notice to industry - removal of requirement for $15 \%$ random replicate samples notice affecting guideline $\mathrm{A}$ and guideline B requirements www.hc-sc.gc.ca/dhp-mps/prodpharma/ applic-demande/guide-ld/bio/15rep-eng.php

103 Garofolo F. The Canadian workshop on the Crystal City AAPS/FDA white paper www.canadianlcmsgroup.com/docs/b

104 Bansal S. Repeat bioanalysis selection and reporting. AAPS Workshop, quantitative bioanalytical methods validation and implementation: best practices for chromatographic and ligand binding assays (2006) www.aapspharmaceutica.com/meetings/ files/64/bansal.pdf 